\section{$\sum_{\text {CHEST }}^{\text {CLNIC }}$}

${ }^{1}$ Department of Pulmonology, St. Antonius Hospital Nieuwegein, Nieuwegein, The Netherlands

${ }^{2}$ Department of Pulmonology, Amphia Hospital, Breda, The Netherlands

${ }^{3}$ Department of Pulmonology, Erasmus MC, Rotterdam, The Netherlands

${ }^{4}$ Department of Radiology, St. Antonius Hospital Nieuwegein, Nieuwegein, The Netherlands ${ }^{5}$ Department of Radiology, Gelderse Vallei Hospital, Ede, The Netherlands

${ }^{6}$ Department of Pathology, St. Antonius Hospital Nieuwegein Nieuwegein, The Netherlands ${ }^{7}$ Department of Pathology, University Hospital Antwerp, Edegem, Belgium

\section{Correspondence to}

CMJ Steendam, Department of Pulmonology, Amphia Hospital, Molengracht 21, Breda 4818 CK, The Netherlands; cmjsteendam@gmail.com

Received 20 November 2016 Revised 12 January 2017 Accepted 13 January 2017

Published Online First 7 February 2017

\title{
Pulmonary artery aneurysms and thrombi
}

\author{
C M J Steendam, ${ }^{1,2,3}$ A L Diederik, ${ }^{4,5}$ A Dendooven, ${ }^{6,7}$ R J Snijder, ${ }^{1}$ G J Herder ${ }^{1}$
}

A 19-year-old previously healthy boy was referred to our hospital with fever, progressive coughing, dyspnoea and haemoptysis for 2 weeks. He had lost weight $(3 \mathrm{~kg})$ and was experiencing night sweats.

In the past 6 months, he had experienced that his overall condition and training capacity in sports had decreased noticeably.

On examination, he was tachypnoeic and tachycardic with normal blood pressure. Oxygen saturation was $90 \%$ with $15 \mathrm{~L}$ of oxygen delivered by Venturi mask. There were diminished breathing sounds in the basal segments of the right lung and in the upper paravertebral region. No other abnormalities were identified.

The laboratory results included a mild microcytic anaemia, leucocytosis and elevation of $\mathrm{C}$ reactive protein.

CT and conventional studies showed a central mass in the right upper lobe (figure 1A, B).

Positron emission tomography-CT (PET-CT) and magnetic resonance angiography (figure 1C) demonstrated (pseudo-) aneurysms of the pulmonary arteries in both lungs, ranging in size from 1 up to $6 \mathrm{~cm}$ in diameter, with thrombus lining the walls and in the central pulmonary arteries. The vascular walls showed enhancement after gadolinium admission and fludeoxyglucose (FDG) uptake.

There were no lesions found elsewhere in the body.

The differential diagnosis of pulmonary artery aneurysms includes infection (eg, TB, syphilis, bacterial, viral or fungal), structural cardiac abnormalities, pulmonary hypertension, idiopathic vasculitis syndromes (Hughes-Stovin syndrome and Behçet's disease), trauma and miscellaneous. ${ }^{12}$

There were no signs of structural cardiac abnormalities, pulmonary hypertension, trauma, infection, neoplasm, autoimmune disorders causing vasculitis or signs of Behçet's disease. We diagnosed Hughes-Stovin syndrome after consultations at a multidisciplinary team meeting and with international experts.

We started immunosuppressive therapy with methylprednisolone (1000 mg a day, during 3 consecutive days) and cyclophosphamide $\left(750 \mathrm{mg} / \mathrm{m}^{2}\right)$. After the first cycle, the patient developed signs of infection, for which broad-spectrum antibiotics was given in combination with prednisone maintenance therapy.

Follow-up FDG-PET-CT showed progression, and his clinical condition deteriorated with progressive haemoptysis. We performed extensive embolisation of the aneurysms without success. During the procedure, pulmonary artery pressure was $65 / 31 \mathrm{~mm} \mathrm{Hg}$ (mean $44 \mathrm{~mm} \mathrm{Hg}$ ) with normal wedge pressure $(7 \mathrm{~mm} \mathrm{Hg})$.

The patient was admitted to the intensive care unit, where he developed respiratory failure and exhaustion. The patient died approximately 6 weeks after admission.

The autopsy revealed multiple bilateral aneurysms and thrombi. Although not macroscopically evident, microscopic examination revealed that the thrombi were composed of a pleomorphic, intravascularlocated tumour process consistent with high-grade pulmonary intima sarcoma (figure 2).

\section{DISCUSSION}

Highes-Stovin syndrome is a rare vasculitic disorder of unknown aetiology. As such, it does not yet have formally described diagnostic criteria and is a diagnosis of exclusion. This disorder induces thrombophlebitis, followed by the formation of large pulmonary and/or bronchial artery aneurysms, and eventually leads to aneurysmal rupture with massive haemoptysis and death. ${ }^{2}$

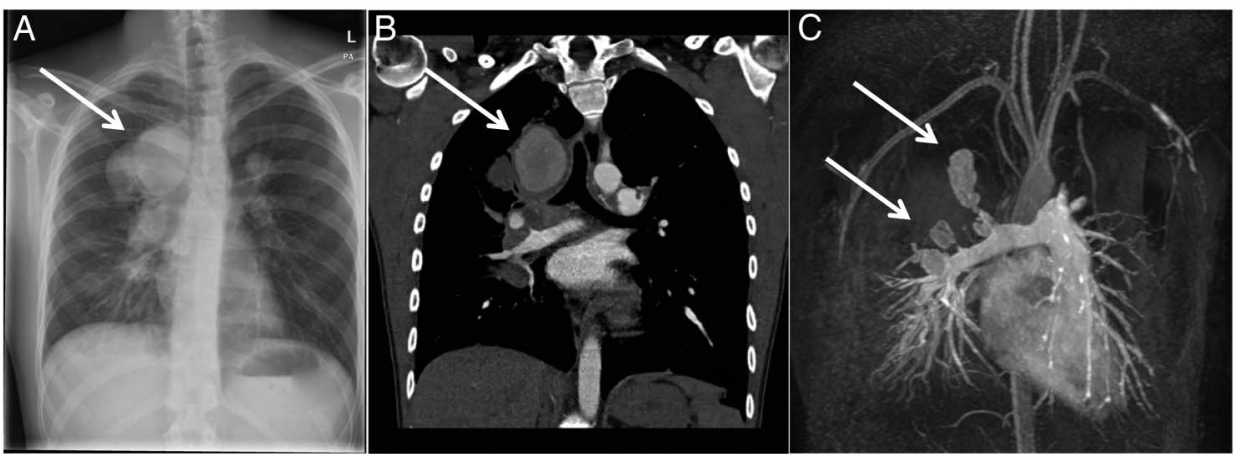

Figure 1 (A) The mass in the right upper lobe on conventional posterior-anterior study, (B) on coronal reconstruction of CT. (C) Magnetic resonance angiography showing the aneurysms in coronal reconstruction. 
Figure 2 (A) The macroscopic view of the right lung from the hilus with evident aneurysms and thrombi. (B) The microscopy with growth of the tumour in the lumen of a branch of the pulmonary artery and pulmonary angiosarcoma (H\&E stain).

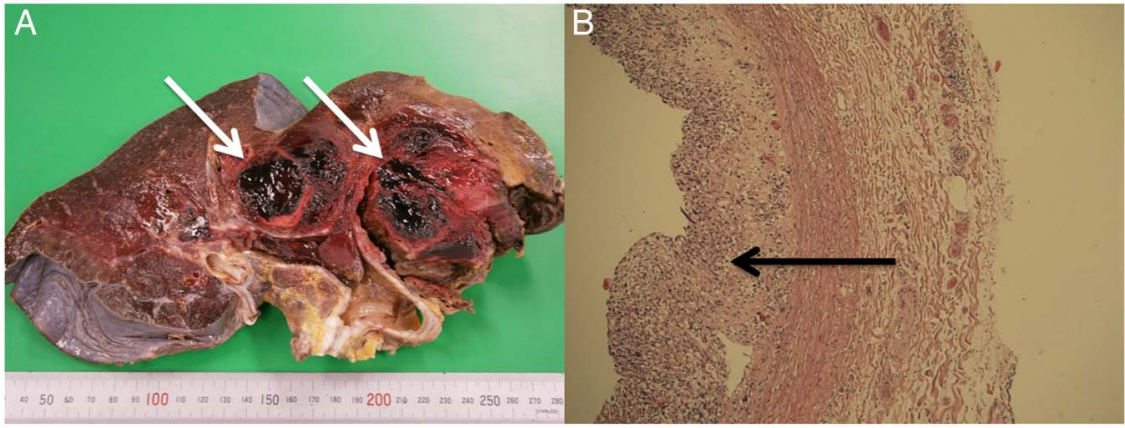

Pulmonary artery sarcoma is a rare disease. ${ }^{3}$ It is known to resemble thromboembolic disease, but this aneurysmatic presentation is even more rare. ${ }^{4}$

This case shows how difficult diagnosis on pulmonary artery aneurysms is in an unstable clinical condition and confirms the poor prognosis of pulmonary angiosarcoma as described in literature.

Contributors All authors wrote, revised and approved the final manuscript. Competing interests None declared.
Patient consent Obtained.

Provenance and peer review Not commissioned; externally peer reviewed.

\section{REFERENCES}

1 Theodoropoulos $\mathrm{P}$, Ziganshin BA, Tranquilli M, et al. Pulmonary artery aneurysms: four case reports and literature review. Int J Angiol 2013;22:143-8.

2 Khalid U, Saleem T. Hughes-Stovin syndrome. Orphanet J Rare Dis 2011;6:15.

3 Blackmon SH, Rice DC, Correa AM, et al. Management of primary pulmonary artery sarcomas. Ann Thorac Surg 2009;87:977-84.

4 Terra RM, Fernandez A, Bammann RH, et al. Pulmonary artery sarcoma mimicking a pulmonary artery aneurysm. Ann Thorac Surg 2008;86:1354-5. 